\title{
UNA NUEVA CONTRIBUCIÓN A LA TÉCNICA DE LA MÚSICA DE TECLA ANTIGUA IBÉRICA: UNAS DIGITACIONES PARA ÓRGANO DE 1649
}

\author{
Miguel Bernal RiPOLL
}

\begin{abstract}
Exposition and comentary of organ fingerings from an anonymous treatise published in Madrid in 1649 (attributed to Tomás Gómez in Lorente's "El porqué de la Música"), denoting their similarity with the fingerings put forward by Hernando de Cabezón in 1578. Other interesting points made in this treatise are also summarized: instructions for choir conduction, seven syllable solfa without mutations, proportions. The treatise also shows the prevailing use of spanish organ tablature ("cifra") in the seventeenth century, as well as the significance of Cabezon's works almost a hundred years after being printed.
\end{abstract}

\section{Resumen}

Se exponen y comentan unas digitaciones para órgano de un tratado anónimo publicado en Madrid en 1649 (atribuido a Tomás Gómez por Lorente en «El porqué de la Música»), señalando su similitud con las digitaciones propuestas por Hernando de Cabezón en 1578. Se resumen otros detalles de interés de dicho tratado: instrucciones para el gobierno del coro, solfeo con siete sílabas y sin mutanzas, proporciones. El tratado muestra también la vigencia del sistema de notación por cifra en el siglo XVII, así como la importancia de la obra de Cabezón casi cien años después de su publicación.

Estudiando los antiguos tratados de Canto de Órgano, en busca de datos que aclaren los problemas de interpretación que presenta la música antigua ibérica para tecla, hemos encontrado una breve referencia a la técnica manual del órgano que hasta ahora parece haber pasado inadvertida, y que debe ser incluida en el repertorio de referencias históricas sobre el toque y las digitaciones.

Se trata de un breve apunte que cierra el tratado anónimo «Arte de Canto Llano, Órgano y Cifra», publicado en Madrid en 1649, y que hasta ahora no había suscitado gran atención entre los investigadores ${ }^{1}$. En ese libro, además de tratarse las cuestiones esenciales de Canto Llano y

1. Conozco la existencia de este tratado gracias a una bibliografía sobre la música española del siglo XVII suministrada por José Vicente González Valle. La existencia de estre tratado en la Biblioteca Nacional había sido consignada por Barbieri y más recientemente por Francisco José León Tello. También Antonio Ezquerro lo cita en la bibliografía de su tesis doctoral. 
Canto de Órgano, y de explicar la cifra de tecla (la misma empleada por Bermudo, Venegas, Cabezón o Correa), hay un breve apunte sobre la técnica manual del órgano, con unas instrucciones para la digitación que son el principal objeto de este artículo. Pese a su brevedad, y a ser casi una copia de las expuestas por Hernando de Cabezón, tienen el interés de representar las únicas digitaciones encontradas en España desde las que recomienda Correa de Araujo en el prólogo de su Facultad Organica (1626) hasta las descritas por Nassarre en Escuela Musica (1724) En efecto, hasta el momento no habían aparecido otras indicaciones de digitación en esta época central del siglo XVII, en la que florecieron tantos grandes organistas. Hay que consignar también el hecho de que se hable por primera vez de digitaciones y técnica únicamente para el Órgano, pues aunque está fuera de duda la equivalencia en la técnica de todos los instrumentos de tecla de la época, hasta el momento se hacía siempre referencia al monacordio como instrumento primario para el estudio ${ }^{2}$.

Otros aspectos del mencionado tratado merecerán también al menos un breve comentario, por su gran interés y novedad.

\section{Autoría del tratado}

El tratado es publicado en Madrid en 1649, y, según aclara el prólogo titulado La Religión a sus prelados y súbditos, está concebido como un manual práctico para el uso de los religiosos de la orden de San Bernardo, con el ánimo de asegurar la calidad de la música en el Culto Divino. El autor permanece anónimo, quizá de forma voluntaria y en ejercicio de humildad monacal. Censura, Aprobación y Licencia hacen referencia al libro compvesto por un monge de la Orden de San Bernardo, sin desvelar la identidad de su autor. Dichas Censura, Aprobación y Licencia son respectivamente de Juan Verjón ${ }^{3}$, Catedrático de Música de la Universidad de Salamanca, Fray Germán de la Serna, Trinitario de Madrid, calificado de «músico insigne», y Alonso de Morales Vallesteros, Canónigo de la Catedral de Toledo y Vicario General de la Villa de Madrid (este último hace sólo la censura moral).

Es Andrés Lorente, en su tratado El porqué de la Música, quien nos desvela la identidad del autor. En efecto, al tratar del canto sin mutanzas dice que sobre este tema «hizo vn Tratado vn Religioso del Orden de Nuestro Padre S. Bernardo (cuyo nombre es, el P. Fr. Tomas Gomez, el año de 1649, sacandole à luz por cosa nueua...». Saldoni parece haberse basado en Lorente

2. Sancta Maria habla de "poner las manos en las teclas del Monacordio» (Arte de tañer fantasia I, f. 37 v.) Venegas y Cabezón dirigen sus consejos a los «instrumentos de Tecla». La Facultad Organica de CORREA DE ARAuXo, aunque está dirigida al órgano, presupone el uso del monacordio como instrumento de estudio.

3. "VERJON de la Real, P. Fr. Juan: maestro en artes. En 1646 fué nombrado catedrático de música de la Universidad de Salamanca, cuya plaza desempeñó hasta 1669, en que se jubiló: su antecesor en dicha plaza fué el P. Fr. Roque Martínez, y su sucesor el P. Fr. Antonio de Castro". Baltasar SALDoni, Diccionario Biográfico-Bibliográfico de Efemérides de Músicos Españoles. Madrid, 1881.

4. A. Lorente. El porqué de la Música. Alcalá de Henares, 1672. f. 50. 
para atribuir también a Tomás Gómez este tratado, ofreciéndonos además algún dato biográfi$\mathrm{co}^{5}$.

\section{Contenido del tratado} siguiente:

El libro, de tan sólo veintidós folios, se divide en cuatro capítulos, cuyo contenido es el

- Capítulo I: Arte de cantar con mutanzas. explica brevemente pero de forma muy práctica y clara los rudimentos suficientes para dominar el canto llano: voces (sílabas de solmisación: ut, re, mi, fa, sol, la), mutanzas, signos (notas absolutas: Csolfaut, Dlasolre, Elami, Ffaut, Gsolreut, Alamire, Bfabmi), deducciones, claves, movimientos (intervalos) y tonos (los ocho clásicos, con bemol en la clave para los tonos $5^{\circ}$ y $6^{\circ}$, de forma similar a lo formulado por Sancta Maria $\left.^{6}\right)$.

También encontramos al final del capítulo unas advertencias para el gobierno del coro, que de modo resumido serían las siguientes: Hay diferentes velocidades para el canto según el carácter del día o la hora. Es mejor no turbar la fluidez y regularidad del compás ni siquiera para intentar enmendar un tempo inadecuado. El Presidente (oficiante de la liturgia) no debe intervenir de forma brusca para intentar cambiar el ritmo de la música cuando le parezca inadecuado, ni siquiera debe llevar el compás: es una responsabilidad que debe dejarse al Cantor (maestro del coro), y en cualquier caso «llamar al Cantor, ò llegarse a el, y dizirle por seña, ò en voz baxa al oydo, que pique, ò detenga el choro» (f. 8 v.). Estos términos de «picar» o «detener» al coro nos hacen suponer que quizás habría gestos específicos para realizar estas funciones, constituyendo ya una incipiente técnica directorial ${ }^{7}$. El compás se lleva con una vara de seis palmos, que sirve también para señalar en el libro de coro lugares donde debe detenerse el canto según lo requiera el oficio.

- Capítulo II: Arte de cantar sin mutanzas. En este capítulo, sorpresivamente para su época, propone el anónimo monje nada menos que un solfeo basado no en las seis sílabas tradicionales, sino en siete. Para ello añade una séptima sílaba: «ni», (equivalente a nuestra nota «si»). Esta innovación la atribuye a Fray Pedro de Ureña $^{8}$, y la defiende diciendo que no tiene sentido

5. “Gómez, P. Fr. Tomàs: religioso de San Bernardo: en 1649 publicó un tratado sobre las mutanzas que segun el debian usarse en aquella época, poniendo ni, hoy dia si de nuestra escala musical, en lugar de bi que fijaron otros autores anteriores y posteriores al P. Gomez. Creemos ser este Gomez el que citamos en nuestras Efemérides publicadas en Madrid en 1860, y del cual decimos, en la pàgina 173 y siguiente, lo que ponemos a continuación: "Gomez, D. Tomàs: eclesiàstico. Nació en el pueblo de Coca, diócesis de Segovia, en Castilla la Vieja. Adquirió renombre por sus grandes conocimientos en teología y en filosofía. Murió en Barcelona en 1668. Se le atribuye un tratado de canto llano que lleva por título: Reforma del canto llano. No hemos podido adquirir ningún ejemplar de dicha obra, que creemos no ha llegado a imprimirse". Baltasar SALdoni, Diccionario Biográfico-Bibliográfico de Efemérides de Músicos Españoles. Madrid, 1881.

6. Sancta Maria, Arte de tañer fantasia I, «Capítulo xxiiij. De los ocho Tonos generales» f. 60. el compás.

7. En tal caso sería un dato en contra de la opinión de que en esta época la técnica directorial se limitaba a llevar

8. “UREÑA, D. Pedro: fraile español, ciego de nacimiento. Nació en la segunda mitad del siglo XVI. Fué, como ciego, gran pensador, y en 1620 escribió una obra en que proponía que fuese abandonado el sistema de las mutanzas atribuido a Guido de Arezzo, añadiendo á los nombres de las seis primeras notas la sílaba ni, que andando el tiempo fué el si 
el que las voces sean seis habiendo siete notas en la octava. Aduce además la ventaja que supone la eliminación de las mutanzas, siempre difíciles. Se apoya también en su defensa aludiendo a la antiguedad de este solfeo heptáfono, vigente hasta que Guido introdujo el hexacordo, y da incluso la noticia de que «en Santa Clara de Villa-Frechos, dos leguas de Medina de Rioseco, se ha tenido hasta estos tiempos este modo de cantar por las letras de los signos, y en lugar de ut re mi fa sol la, pronuncian $\mathrm{Ge}, \mathrm{A}, \mathrm{Be}, \mathrm{Ce}, \mathrm{De}, \mathrm{E}, \mathrm{Fe}$ : lo qual, segun las señoras Monjas dizen, ha venido por tradicion de vnas a otras, desde q el monasterio se fundò, sin noticia del Autor.» (f. $14 \mathrm{v}$.) Según el nuevo sistema propuesto por el anónimo tratadista, las propiedades se reducirían a dos: bemol y bequadrado (corresponden a las transposiciones de nuestra escala mayor en do o fa), y llega incluso a proponer eliminar la propiedad por bemol, y reducir todo a una sola propiedad. Las voces son siete: ut re mi fa sol la ni. Los signos son Ffaut, Gsolre, Alami, Bfabni, Csolut, Dlare, Enimi (al haber solo dos propiedades, cada signo tiene sólo dos voces). Los tonos son los mismos que en el canto tradicional con mutanzas.

El canto sin mutanzas había sido ya propuesto por Cerone 9 , y veintitrés años más tarde Andrés Lorente lo explica también en El porqué de la Música $(1672)^{10}$, utilizando la sílaba «Bi» en lugar de «ni». Lorente refiere que este sistema ya ha sido explicado por Cerone "y otros», y cita también el tratado de 1649, desvelando incluso la identidad del autor, como hemos señalado más arriba.

Señalemos que mientras Lorente lo explica con una actitud enciclopédica y teórica, el fraile anónimo es defensor y promotor de este sistema. Además el fraile madrileño parece no saber que Cerone había expuesto ya este sistema, señalando como inventor del mismo a Fray Pedro de Ureña, y frente a la afirmación de Lorente de que "no està puesto en vso el modo de cãtar sin mutanças» señala el caso de las monjas de Villa-Frechos. El mismo Lorente más adelante lo califica con la expresión menos excluyente de "poco vsado». Quizás iba surgiendo de forma simultánea en varios lugares una concienza de lo que hoy es la escala mayor, cuestión apasionante a investigar que queda fuera de este breve artículo.

- Capítulo III: Arte de canto de órgano. También de forma muy breve y práctica se exponen los rudimentos de la música mensural: compás, figuras y tiempos, de los que consigna sólo los más usuales: Perfecto Mayor (Ø), Perfecto Menor (O), Imperfecto Mayor (ф), Imperfecto Menor o Compasillo (C) y los de Proporción (que también llama Ternarios): Proporción Menor (C3) y Proporción Mayor (Ø3/2). Todos estos tienen los valores habituales que suponemos bien conocidos por el lector, siendo la única diferencia las señales indiciales con que indica las proporciones, que son por cierto los mismos que utiliza Correa en su Facultad Organica.

\footnotetext{
que hoy usamos, y que inmortalizó el nombre del flamenco que en el mismo siglo XVI inventó esta última sílaba. No creemos tan grande la diferencia de ni á si, para que se prive á nuestro compatriota de la primacía en esta invención, admitiendo como más conveniente la del flamenco". Baltasar SALDONI, Diccionario Biográfico-Bibliográfico de Efemérides de Músicos Españoles. Madrid, 1881.

9. P. CERONE, El Melopeo y maestro, f. 514.

10. A. Lorente. El porqué de la Música. Alcalá de Henares, 1672. ff. 49-55. Capitvlo trigesimo octavo. Modo de Cantar sin Mutanças.
} 
- Capítulo IV: Arte de Cifra. En este breve y último capítulo se explica la notación de la música de tecla en cifra. En esto no se aparta un ápice de lo expuesto por Juan Bermudo, Luys Venegas de Henestrosa, Hernando de Cabezón y Francisco Correa de Araujo, a excepción de la importante novedad de ampliar el ámbito hasta C5 (lo que supone una extensión habitual de 45 notas para el órgano). Como vemos, en esta época este tipo de notación goza de plena salud y vigencia, y al parecer se mantuvo todavía durante todo el siglo, pues Lorente prometió en El porqué de la música un libro de música de órgano en cifra titulado Melodías Músicas que desgraciadamente no ha llegado hasta nosotros, y que quizá no llegó a ver la luz ${ }^{11}$.

Recordamos brevemente las bases de este sistema de notación, que será bien conocido por el lector familiarizado con este tipo de música: Signos Regraves (C1-E1): 567 con dos «rasguillos» ${ }^{12}$. Graves (F1-E2): 1-7 con un «rasguillo». Agudos (F2-E3): 1-7 sin nada. Sobreagudos (F3-E4): 1-7 con punto. Reagudos (F4-C5): 1-5 con vírgula. Los sostenidos y bemoles después de la cifra indican que se toca la nota negra inmediatamente superior o inferior.Una vírgula sirve de ligadura, y un trazo de pausa. Un arco con un 3 sobre un compás indica tocar las figuras en proporción sexquialtera (3 contra 2 de igual valor, o sea, nuestro tresillo). El sistema consta de una serie de líneas paralelas, una por cada voz, en la que se escriben las cifras correspondientes a las notas. Unas rayas verticales delimitan los compases. El ritmo lo indica la posición relativa de las cifras en el espacio que ocupa un compás, o bien es indicado con figuras encima de las cifras. El autor da una tabla con un cierto número de ejemplos de cómo se indican las figuras y los ritmos más comunes, copiada casi íntegramente de la expuesta por Cabezón ${ }^{13}$. Los signos B ó Łal principio de la obra indican la propiedad, es decir, si el signo Bfabmi es bemol o bequadro.

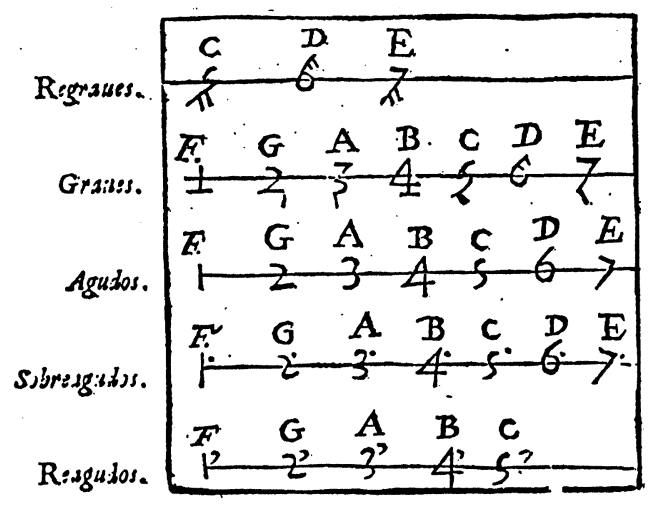

f. 20 v. Los Signos y su correspondencia con las cifras

11. A. LoRente. El porqué de la Música. Alcalá de Henares, 1672. f. 18.

12. El 5 con dos rasguillos sería el Do más grave del teclado del órgano.

13. Hernando de Cabezón, Obras de Mvsica.... "Declaracion de la cifra que en este libro se vsa» f. 4. 


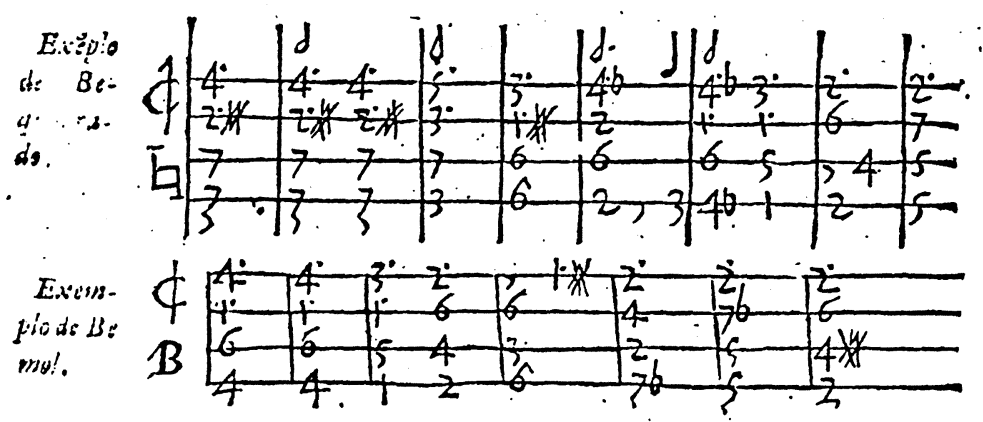

\section{f. 22 v. Ejemplos por Becuadro y por Bemol (transcritos en esta misma página)}

El autor copia literalmente en f. 22v. unos ejemplos por Bemol y por Bequadrado del libro de Cabezón, que son transcritos a continuación. ${ }^{14}$
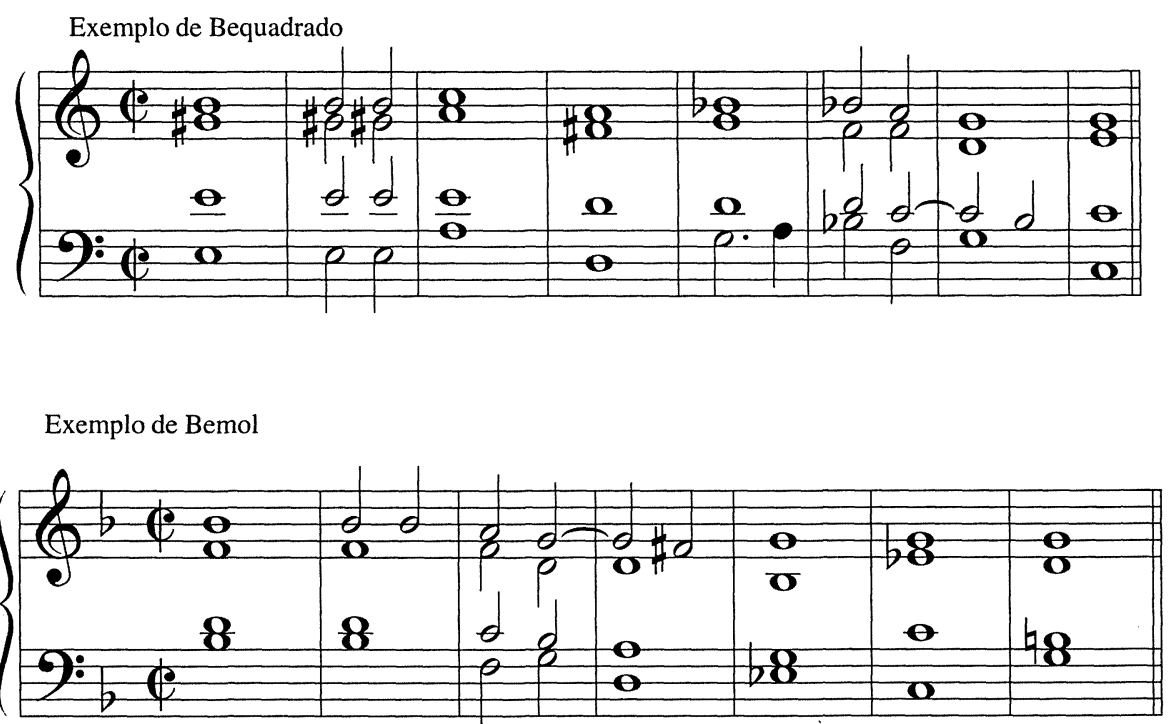

Hacemos la observación de que en estos ejemplos la señal indicial es de Compás Mayor 屯, aunque las barras de compás están cada semibreve como ocurre también en las obras de Cabezón y Correa que están escritas con este tiempo. Una muestra más de que el espacio entre dos barras (y que se denomina normalmente «casa») no siempre coincide con el Compás métrico y menos con el rítmico, y una muestra también de cómo el Compás Mayor acaba por ser el mismo que el Compasillo pero más veloz.

14. Hernando de CABezón, Obras de Mvsica.... «Declaracion de la cifra que en este libro se vsa» f. 4 v. 


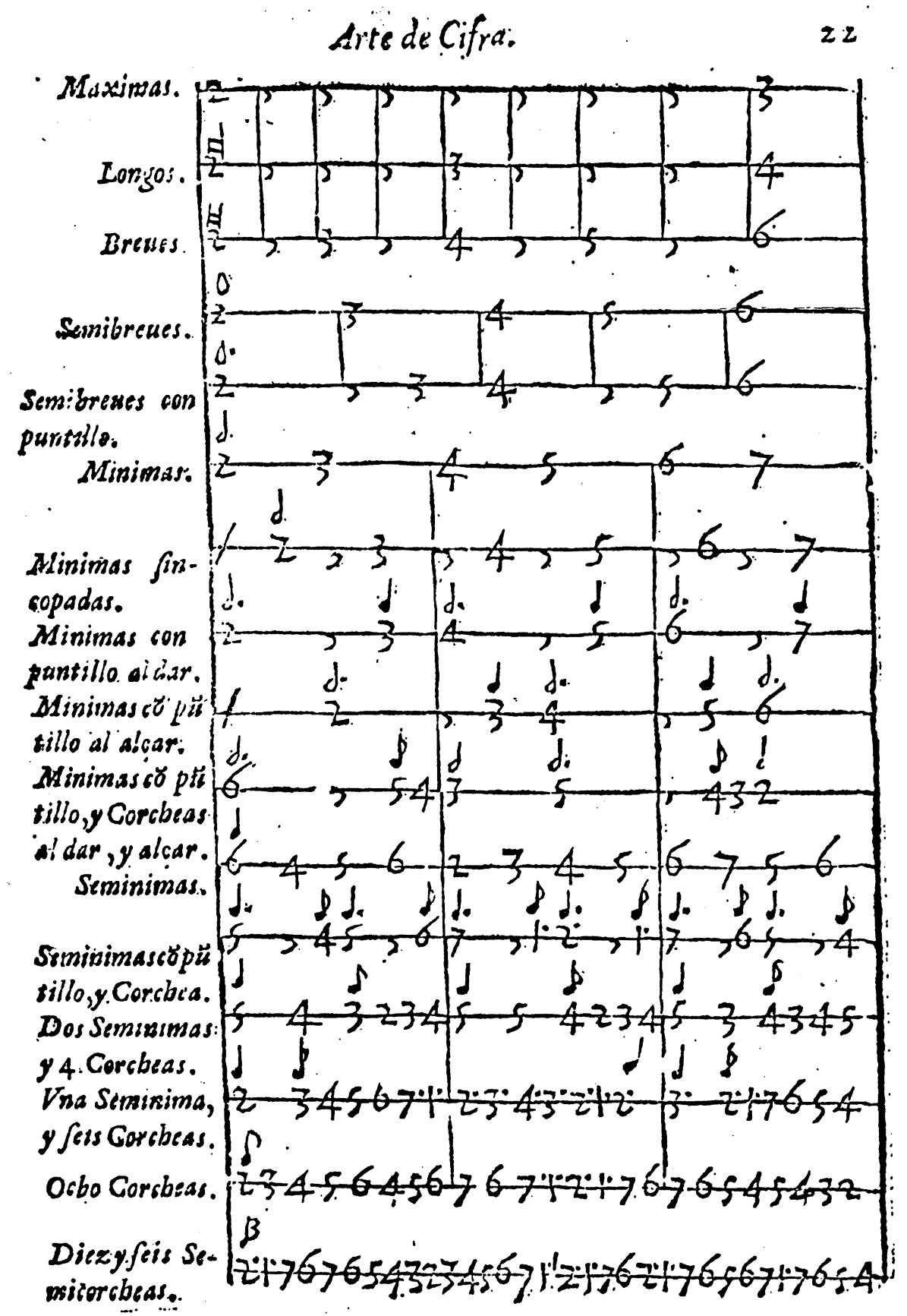

f. 22. Ejemplos de indicación de los ritmos

Anuario Musical, 55 (2000) 


\section{Digitaciones de Madrid, 1649}

Es al final del último capítulo,en un apunte que ocupa el reverso del último folio del libro, donde encontramos unas concisas normas principalmente para la digitación, que serán transcritas en su totalidad y comentadas a continuación. Dar a conocer estas nuevas digitaciones a los organistas interesados en nuestra música antigua es el principal objeto de este artículo.

«Modo de poner las manos en el Organo. §.IV.

1 Ninguna explicacion suple del todo la enseñança viua del Maestro; pero con pocas liciones, buenos deseos, y tocar limpio (esto es no pisar dos teclas juntas con un dedo) valdran estos auisos.

2 Ambas manos tocan la Octaua con Index y Polex. Quartas, Quintas y Sextas con Polex y Quarto ò con Index y Quinto. Terceras con Polex y Medio, con Index y Quarto, ò con Medio y Quinto, como mejor acomodaren para Vozes, y Glossas.

3 La derecha comiença a subir desde el Polex al Quarto; y sin boluer al Polex ni al Index, corre con medio y Quarto. Comiença a baxar con Quarto hasta Index, y sin boluer al Quarto corre con Index y Medio. La izquierda comiença a subir de Quarto a Polex, y repitiendo este circulo, corre su Glossa. Comiença a baxar de Polex à Quarto, y in boluer a Polex ni a Index, corre con Medio y 4.

4 La derecha haze quiebros con Medio y Quarto, con Index y Medio, ò con Index y Polex. La izquierda, con Index y Polex, o con Index y Medio; y ambas hazen siempre la mayor fuerça en la tecla sustenida, que señala la letra.

5 La Cifra impressa de Antonio Cabezon tiene Duos, y Tercios para principiantes, y Obras de primor, para hazer consumados Organistas. Importaria mucho al seruicio de Dios, que la huuiesse en todos los Monasterios, ò papeles de cifra equiualentes.

LAVS DEO» (f. 22v.)

Ante todo, el autor hace una importante advertencia: hay que «tocar limpio (esto es no pisar dos teclas juntas con un dedo)» ${ }^{15}$. A continuación, pasa a proponer las siguientes digitaciones:

- Para los intervalos armónicos (ambas manos):

Octava: 2-1 (esto es seguramente un error, y quiera decir 5-1)

Cuartas, quintas y sextas: 1-4 ó 2-5

Terceras: 1-3, 4-2, 3-5 «como mejor se acomodaren para Vozes, y Glossas».

Destacamos la «modernidad» del 3-5 en las terceras, que ya había expuesto Hernando de Cabezón adelantándose 138 años a Couperin.

- Para las escalas:

Mano derecha, escala ascendente: $1234343434 \ldots$

Mano derecha, escala descendente: $4323232 \ldots$

Mano izquierda, escala ascendente: $432143214321 \ldots$

Mano derecha, escala descendente: $1234343434 \ldots$

15. Quizás del ámbito técnico de la limpieza en la ejecución podemos sin duda extrapolar al ámbito musical de la articulación, recordando la cuarta condición que Sancta Maria formula para «tañer con perfeccion y primor», a saber, "tañer con limpieça y distinction", para la cual Hay que levantar un dedo antes de que hiera el otro, para que no suene "sucio y estropajoso» (SANCTA MARIA, Arte de tañer fantasia I, f. 36 v. y 38 v.). 
Como es habitual en los españoles, coexisten las digitaciones con pulgar y sin pulgar. Al igual que Venegas ${ }^{16}$ y Cabezón, el autor no distingue entre digitaciones más pausadas y más veloces, como hicieron Sancta Maria y Correa. Estos últimos prescribieron para las carreras veloces digitaciones con dedos agrupados por cuatro y utilizando el pulgar. El anónimo fraile se reserva el «paso del pulgar» sólo en la mano izquierda ascendiendo, como Cabezón.

- Para quiebros:

Mano derecha: 34, 23, 12

Mano izquierda: 21,23

El autor no explica sin embargo qué quiere decir con el término quiebro. Parece lógico pensar que se trata del quiebro sencillo, de una sóla batida y superior (a pesar de que en mano izquierda da los dedos 23 en ese orden, lo que indicaría un quiebro inferior). Ese tipo de ornamento lo encontramos escrito en notación regular en obras de Cabanilles, y es descrito por Nassarre como aleado ${ }^{17}$. Recordemos además que Hernando de Cabezón, autor en quien se basa el autor y cuya obra recomienda, describe tan sólo el quiebro superior ${ }^{18}$, mientras Correa describe sólo el inferior ${ }^{19}$. No descartamos en cualquier caso que se trate de una indicación genérica de digitación para trinos tanto cortos como largos.

Es interesante también la observación de que hazen siempre la mayor fuerça en la tecla sustenida, que señala la letra, que se refiere a apoyar más la nota real (aquí tecla sustenida se refiere a la nota principal, no a que esté alterada ascendentemente). Esta precisión puede tener un doble valor técnico y musical: el apoyar esa nota facilita la realización a nivel de mecanismo, y musicalmente indica que la nota principal es más presente en fuerza y valor.

En cualquier caso, dada la brevedad del apunte, podemos suponer que el anónimo autor ha reflejado sólo los preceptos fundamentales. Por ello, el hecho de que hayan incluido, junto a las digitaciones de intervalos y escalas, las de los quiebros, es un claro indicio de la importancia de la ornamentación en este periodo, y otro argumento a favor de que ésta no puede faltar en la interpretación de las obras de nuestros antiguos compositores para tecla. Finalmente no es tan descabellada esa profusión de quiebros que recomiendan nuestros antiguos organistas ${ }^{20}$.

16. Las digitaciones que propone Venegas son las siguientes: Mano derecha: Ascendente: 1234343434 ó 23434 ó 3434. Descendente: 54321321321 ó 4321321321 Mano izquierda: Ascendente: 4321321321 ó 212121 Descendente: 1234343434. Estas digitaciones valen también para la velocidad (... hasta hazerlo de corcheas muy limpio...»). No obstante, estas instrucciones tienen un carácter fundamentalmente pedagógico: «... entiéndase esto para los principiantes, que después como se offreciere el paso, así pondrá los dedos, como lo haga con más facilidad.» (Luys VENEGAS DE HENESTROSA. Libro de Cifra Nueva. ff. 7v.-8. Alcalá de Henares, 1557).

17. Ver nuestro trabajo «La ornamentación en las obras de Cabanilles» en Anuario Musical 53, 1998.

18. Dice Hernando textualmente «quiebren de la parte de arriba» (HERNANDO DE CABEZÓN, Obras de Mvsica.... f. 7).

19. F. Correa De Arauxo, Facultad Organica, Prólogo en alabança de la cifra, f. $15 \mathrm{v}$.

20. "Los Qyebros reyterados se hazen en todas las Minimas, que se hieren con dedos que se pueden hazer, mas los Senzillos no se hazen en todas las Seminimas, pero en la vna si, y en la otra no, y desta manera todas» (Tomás de SANCTA MARIA, Arte de tañer fantasia. Valladolid, 1565). "se ha de quebrar com a mão esquerda, \& direita, todas as vezes que fos possivel» (Manoel Rodrigues Coelho, Flores de Musica. Lisboa 1620). "Si fuere musica totalmente llana (o muy gran parte) la aveis de adornar con estos accidentes [quiebros]; y assi en todos los golpes de semibreves, y minimas, podeis hazer uno de ellos (conforme esta declarado) y parecera bien dexar algunos puntos llanos, de quando en quando sin ellos» (Francisco Correa de Arauxo. Facultad Orgánica. Alcalá, 1262. Prologo, f. 16v.). 
Seguramente el lector familiarizado con la técnica del órgano histórico habrá descubierto ya que el monje no ha hecho sino copiar casi al pie de la letra las instrucciones que para la digitación da Hernando de Cabezón, y que transcribimos a continuación para poder apreciar su similitud:

«EL ORDEN QVE SE HA DE TENER PARA subir y baxar en la tecla.

Con la mano derecha han de subir con el tercero y quarto dedo, y baxar con el tercero y segundo, contando desde el pulgar que es el primero. Con la yzquierda han de subir con el quarto, y yr consecutiuamente hasta el primero, y luego tornar al quarto, y asi vaya subiendo todo lo que quisiere. Ha de baxar desde el pulgar hasta el quarto, y despues yr baxando con tercero y quarto hasta donde quisiere.

Las sextas y quintas ansi con la mano derecha como con la yzquierda, han de dar cõ primero y quarto, con primero y tercero dedos, terceras se dan con quarto y segundo, y primero y tercero, y tercero y quinto dedos. Esto se supone para los que no saben ninguna cosa tañer, y aduiertan que nunca den dos teclas con vn dedo, y tengan mucha quenta de tañer muy limpio lo que pusieren, y hasta que no tañan una obra muy a compas y sin errar, no pongan otra, que sera trabajar en vano, y despues toparan glosas que no se podra tener esta orden de dedos, cada vno las haga con los dedos que mejor se amañare.

Los quiebros se han de hazer con la mano derecha, con tercero y quarto, y con segundo y tercero dedos, y con la mano yzquierda, con tercero y segundo, y con segundo y primero dedos, y quiebren de la parte de arriba lo mas apriesa que pudieren, y no ha de ser largo, sino lo mas corto que pudiere, haziendo siempre mas fuerça en la tecla que la figura de la cifra demonstrare, donde a el le pareciere hazer quiebro». ${ }^{21}$

Observamos que el tratado anónimo de 1649 repite casi literalmente algunas de estas indicaciones de Cabezón, como la recomendación de «tañer con limpieza», y el «hacer fuerza» en la nota principal de los quiebros. En las digitaciones sólo se aparta el anónimo fraile muy levemente de lo expuesto por Cabezón, a saber:

- El tratado de 1649 da una digitación para las octavas (que seguramente es errónea, como hemos señalado).

- En las cuartas y quintas prescribe 1-4 ó 2-5 frente a 1-4 ó 1-3 de Cabezón.

- Las escalas en mano derecha Cabezón las da como alternancia de dos dedos, mientras que el tratadista anónimo comienza la descendente en mano derecha con cuarto dedo: 4323232 etc. y la ascendente con primero: $12343434 \ldots$ En esto se asemeja más a Venegas (ver nota 16), cuya obra quizás conocería también el autor.

- Se da también la posibilidad de 1-2 en mano derecha para los quiebros.

El autor no oculta su fuente, pues el tratado acaba haciendo una mención explícita del libro de Cabezón, recomendando incluso que dicho libro se encuentre en todos los Monasterios. Esta observación, junto al hecho de que en las digitaciones siga fielmente a Cabezón, demostraría la influencia que la obra de éste último tendría todavía en el ámbito musical.

21. Hernando de Cabezón. Obras de Mvsica... Madrid, 1578. Declaracion de la cifra... ff. 6 v.-7 


\section{Conclusiones de carácter general}

En conclusión, de la lectura de este breve pero interesante tratado podemos añadir al estado actual de los conocimientos en praxis interpretativa de la música antigua ibérica unas nuevas instrucciones de digitación. Éstas se caracterizan por estar basadas principalmente en las de Hernando de Cabezón, y también en alguna medida en Venegas. Una característica de las digitaciones expuestas, tanto las de Cabezón de 1578 como las anónimas de 1649, es la combinación de esquemas «antiguos» (escalas con dedos agrupados por dos y sin pulgar, intervalos armónicos siempre con mismos dedos) y «modernos» (escalas con dedos agrupados por cuatro utilizando el pulgar, terceras con 35). En cualquier caso, el autor está más cerca de planteamientos «antiguos» descritos por Venegas y Cabezón, no llegando a plantear el uso generalizado del pulgar en las escalas veloces, como hicieron Sancta Maria y Correa. La falta de originalidad del anónimo autor del tratado no invalida el interés de este hallazgo, que nos indica la vigencia de la técnica descrita por Cabezón y Venegas casi cien años más tarde, al menos con carácter pedagógico en determinados círculos.

Otras consideraciones que se deducen del examen de este anónimo «Arte de Canto Llano, Órgano y Cifra», y que en nuestra opinión son de interés son las siguientes (ordenadas por temas):

Teoría:

- La existencia en España a mitades del siglo XVII del solfeo absoluto con siete notas en lugar de la solmisación guidoniana basada en el hexacordo, al menos en algún caso aislado. Esto nos llevaría a sospechar que ya en esa época hay una conciencia de la escala mayor heptáfona, aunque en modo alguno pretendemos sostener que esto presuponga una conciencia tonal.

\section{Praxis interpretativa:}

- La advertencia de dejar la dirección musical a la responsabilidad del Maestro, que a su vez nos indica que quizás los oficiantes se inmiscuían en ese campo más de lo que los músicos deseaban. En cualquier caso se pone en relieve la necesidad de los músicos de realizar su labor con perfección y profesionalidad, de expresarse según sus propias ideas.

- La necesidad de un pulso regular y sin brusquedades para la música de esta época.

- La posibilidad de que hubiera gestos específicos para ligeros cambios agógicos (picar, detener) aunque no podemos asegurar si era para producir matices agógicos o sólo para corregir errores de tempo sobre la marcha.

- La presencia de los quiebros como elemento fundamental en la ejecución.

\section{Organología:}

- La extensión de 45 notas como usual en el órgano. 
Notación:

- La vigencia de la cifra todavía a mitades del siglo XVII.

\section{Repertorio:}

- La vigencia de la obra de Cabezón todavía a mitades del XVII, como fuente de repertorio y como material pedagógico.

Acabaremos este breve artículo insistiendo en que no debemos dar a las digitaciones antiguas un carácter de dogma. Los mismos autores antiguos, tal como acabamos de leer en Cabezón, insisten en su carácter pedagógico de norma primaria o inicial, para «los que no saben ninguna cosa tañer $»^{22}$, o «para los principiantes ${ }^{23}$ y siempre acaban diciendo que será necesario buscar nuevas digitaciones cuando aparezcan dificultades determinadas, las cuales «cada vno las haga con los dedos que mejor se amañare».

22. Cabezón, ver fragmento transcrito en pág. 92.

23. Ver nota 16. 